\title{
Top Semirelib Graph of a Tree
}

\author{
K. B. Manjunatha Prasad \\ Sri Siddaganga College for Women, \\ Tumkur, \\ Research Scholar, CMJ University, \\ Meghalaya, India
}

\author{
Venkanagouda M Goudar, \\ $\mathrm{PhD}$. \\ Professor of mathematics \\ Dept. of Mathematics \\ Sri Siddhartha Institute of \\ Technology,
}

\author{
Shiva Kumar K B, PhD. \\ Prof, Dept of Telecommunication \\ Sri Siddhartha Institute of \\ Technology, \\ Maralur, Tumkur,
}

\begin{abstract}
In this communications, the concept of top semirelib graph of a planar graph is introduced. We present a characterization of graphs, whose top semirelib graphs are always seperable. Further characterize graph whose plan $\mathrm{T}_{\mathrm{ps}}(\mathrm{T})$ is planar and outer planar. Lastly we proved that $T_{p s}(T)$ is always noneulerian and non Hamiltonian.
\end{abstract}

\section{INTRODUCTION.}

The concept of pathos semirelib graph of a tree $\mathrm{T}$ is found in [5]. Pathos semirelib graph of a tree $\mathrm{T}$ denoted by $\operatorname{Ps}(\mathrm{T})$ is the graph whose vertex set is the union of set of edges, set of blocks, set of regions and the path of pathos of $\mathrm{T}$ in which two vertices are adjacent if and only if the corresponding edges of $\mathrm{T}$ are adjacent, the corresponding edges lies on the blocks, the corresponding edges lies on the region and the edges lies on the pathos.

All undefined terminology will conform with that in Harary [2]. All graphs considered here are finite, undirected, planar and without loops or multiple edges.

We now define the top semirelib graph of a tree. The Top semirelib graph of a tree $T$ denoted by $T_{p s}(T)$ is the graph whose vertex set is the union of set of edges, set of blocks, set of regions and the path of pathos of $\mathrm{T}$ in which two vertices are adjacent if and only if the corresponding edges of $\mathrm{T}$ are adjacent, the corresponding edges lies on the blocks, the corresponding edges lies on the region and the edges lies on the pathos and paths have common edges. We depict the graph $\mathrm{G}$ and its $\mathrm{T}_{\mathrm{ps}}(\mathrm{G})$ in the figure 1 .

The edgedegree of an edge $\{u, v\}$ is the sum of the degree of the vertices of $u$ and $v$. For the planar graph $G$, the inner vertex number $i(G)$ of a graph $G$ is the minimum number of vertices not belonging to the boundary of the exterior region in any embedding of $\mathrm{G}$ in the plane. A graph $\mathrm{G}$ is said to be minimally non-outer planar if $i(G)=1$ as was given by Kulli [4]

\section{Preliminary Notes}

We need the following results to prove further results.

\section{Theorem 2.1 [2]:}

If $G$ is a $(p, q)$ graph whose vertices have degree $d_{i}$ then the line graph $\mathrm{L}(\mathrm{G})$ has $\mathrm{q}$ vertices and $\mathrm{q}_{\mathrm{L}}$ edges, where $\mathrm{q}_{\mathrm{L}}=-\mathrm{q}+1 / 2 \mathrm{~d}^{2}$ edges.

\section{Theorem 2.2 [2]:}

The line graph $\mathrm{L}(\mathrm{G})$ of a graph is planar if and only if $\mathrm{G}$ is planar, $\Delta(\mathrm{G}) \leq 4$ and if $\operatorname{deg} \mathrm{v}=4$, for a vertex $\mathrm{v}$ of $\mathrm{G}$, then $\mathrm{v}$ is a cut vertex.

\section{Theorem 2.3 [3]:}

A graph is planar if and only if it has no sub graph homeomorphic to $\mathrm{K}_{5}$ or $\mathrm{K}_{3,3}$.

\section{Theorem 2.4 [4]:}

A graph is outerplanar if and only if it has no subgraph homeomorphic to $\mathrm{K}_{4}$ or $\mathrm{K}_{2,3}$.

\section{Theorem 2.5 [5]:}

For any non trivial tree $\mathrm{T}, \mathrm{P}_{\mathrm{s}}(\mathrm{T})$ is outer planar if and only if $T$ is a path $P_{n}, n \leq 3$.

\section{Main Results}

We start with few preliminary results.

\section{Lemma 3.1:}

For any tree $\mathrm{T}, \mathrm{L}(\mathrm{T}) \subseteq \mathrm{T}_{\mathrm{ps}}(\mathrm{T})$.

\section{Lemma 3.2:}

If a tree $\mathrm{T}$ contains a block as $\mathrm{K}_{2}$, then it becomes a pendent vertex in $\mathrm{T}_{\mathrm{ps}}(\mathrm{T})$.

In the following theorem we obtain the number of vertices and edges of a Top semirelib graph of a tree

\section{Theorem 3.3:}

For any nontrivial tree $T$, the top semirelib graph $T_{p s}(T)$ whose vertices have degree $d_{i}$ and path number $k$ has ( $q$ $+\mathrm{r}+\mathrm{b}+\mathrm{k}) \quad$ vertices and $\frac{1}{2} \sum d_{i}^{2}+\sum q_{j}+q+\frac{1}{2}\left[n_{i}\left(n_{i}-1\right)\right]$ edges where $r$ and $b$ be the number of regions and blocks respectively and $n_{i}$ be the degree of the star $v_{i}$.

Proof: By the definition of $\mathrm{T}_{\mathrm{ps}}(\mathrm{T})$, the number of vertices is the union of edges, regions, blocks and the path of pathos of $T$. Hence $T_{p s}(T)$ has $(q+r+b+k)$ vertices. Further by the Theorem 7.2.1, 

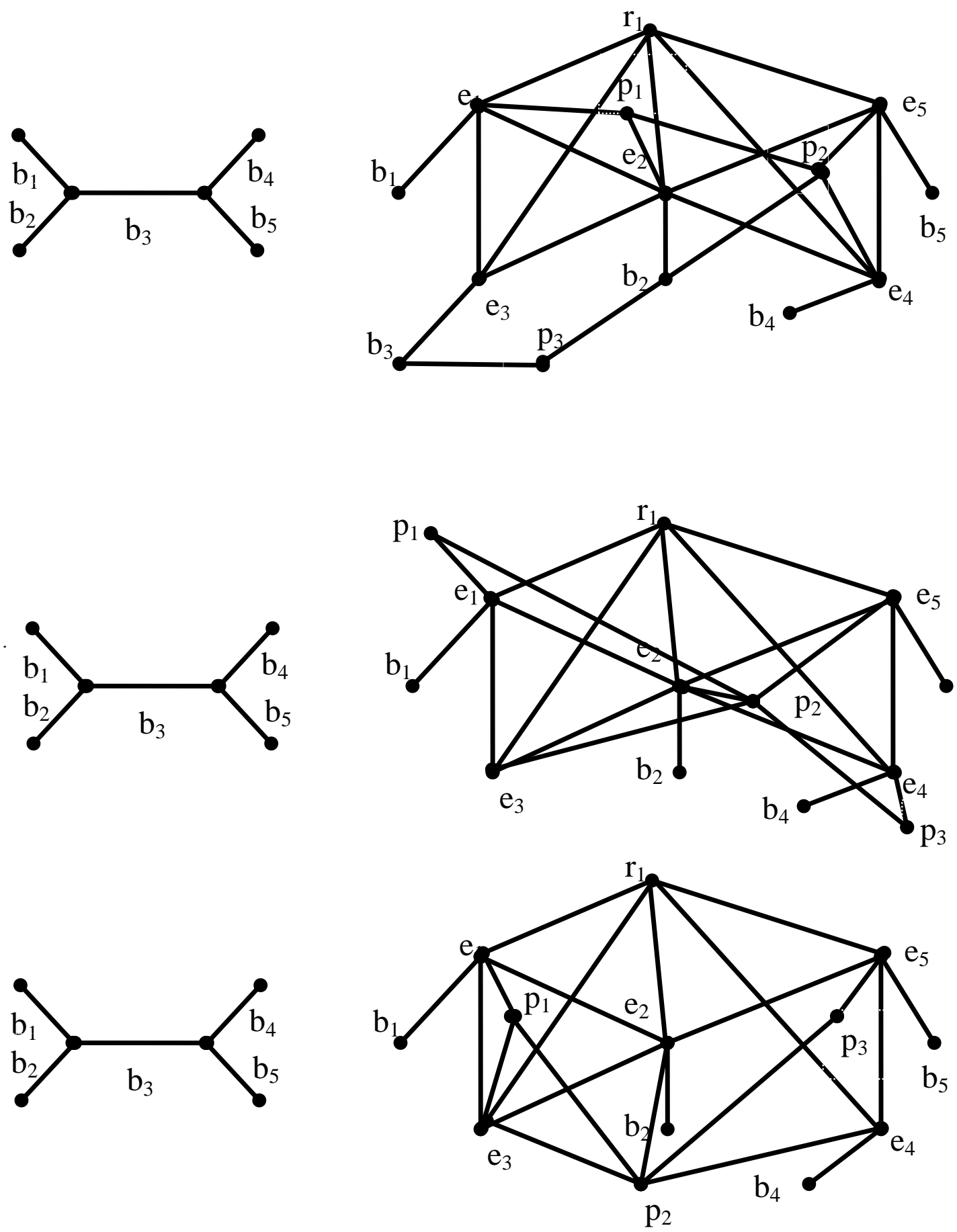

G

Figure 1

$$
\mathrm{T}_{\mathrm{ps}}(\mathrm{G}) \text {. }
$$


$q_{L}=-q+\frac{1}{2} \sum d_{i}^{2}$

number of edges in $\mathrm{L}(\mathrm{T})$ is Thus the number of edges in $T_{p s}(T)$ is the sum of the number of edges in $\mathrm{L}(\mathrm{T})$, the number of edges bounded by the regions which is $\mathrm{q}$, the number of edges lies on the blocks is $\sum q_{j}$ and the number of edges lies on the path of pathos which is $q$ and the number of vertices $n_{i}$ which lies on the pathos $p_{i}$ which is $\left[n_{i}\left(n_{i}-1\right) / 2\right]$. Hence

$\mathrm{E}\left[\mathrm{T}_{\mathrm{ps}}(\mathrm{T})\right]=\frac{1}{2} \sum d_{i}^{2}+\sum q_{j}+q+\frac{1}{2}\left[n_{i}\left(n_{i}-1\right)\right]$.

\section{Theorem 3.4:}

For any edge in a tree $\mathrm{T}$ with edge degree $\mathrm{n}$, the degree of the corresponding vertex in $\mathrm{T}_{\mathrm{ps}}(\mathrm{T})$ is $\mathrm{n}+1$.

Proof: Suppose an edge $e \in E(T)$ have degree n. By the definition of top semirelib graph, the corresponding vertex in $T_{p s}(T)$ has $n-1$. Since edge is a block, we have the degree of the vertex is $n-1+1=n$. Further, the edge lies on a path of pathos. Clearly degree of $e_{i}$ is $n+1$.

\section{Theorem 3.5 :}

If $T$ is a tree with $n$ edges then $T_{p s}(T)$ contains $n$ pendent vertices.

Proof: Let $e_{1}, e_{2}, \ldots e_{n} \in E(T), b_{1}=e_{1}, b_{2}=e_{2}, \ldots b_{n}=$ $e_{n}$ be the blocks and $r_{1}, r_{2}, \ldots r_{k}$ be the regions of $G$. By the definition of line graph $\mathrm{L}(\mathrm{T}), \mathrm{e}_{1}, \mathrm{e}_{2}, \ldots \mathrm{e}_{\mathrm{n}}$ form a subgraph without isolated vertex. Consider that $\mathrm{T}$ does not contain any pendent pathos. By the definition of $\mathrm{T}_{\mathrm{ps}}(\mathrm{T})$, the region vertices are adjacent to these vertices to form a graph without isolated vertex. Since there are $\mathrm{n}$ blocks which are $\mathrm{K}_{2}$, we have each $\mathrm{b}_{1}=\mathrm{e}_{1}, \mathrm{~b}_{2}=\mathrm{e}_{2}$, $\ldots b_{n}=e_{n}$ are adjacent to $e_{1}, e_{2}, \ldots e_{n}$. Hence $T_{p s}(T)$ contains $n$ pendent vertices. If a tree $T$ contains a pendent pathos, then the vertex $b_{i}=e_{i}$ is adjacent to the pathos vertex $P_{i}$ to form a pendent vertex. Since in top semirelib graph of a tree, one path of pathos is adjacent to at least other pathos we have degree of a pathos vertex is at least two. Hence the result follows.

\section{Theorem 3.6:}

For any tree $\mathrm{T}, \mathrm{T}_{\mathrm{ps}}(\mathrm{T})$ is separable.

Proof: Let $\mathrm{e}_{1}, \mathrm{e}_{2}, \ldots \mathrm{e}_{\mathrm{n}} \in \mathrm{E}(\mathrm{T}), \mathrm{r}_{1}, \mathrm{r}_{2}, \ldots \mathrm{r}_{\mathrm{k}}$ be the regions and $b_{1}, b_{2}, \ldots b_{m}$ be the blocks which are $K_{2}$. By the definition of line graph, $\mathrm{e}_{1}, \mathrm{e}_{2}, \ldots \mathrm{e}_{\mathrm{n}}$ form a subgraph without isolated vertex. Since each block is $\mathrm{K}_{2}$, clearly each block of $\mathrm{T}$ contains exactly one edge and each region of $\mathrm{G}$ has at least three edges. If a tree $\mathrm{T}$ contains a pendent pathos, then the vertex $b_{i}=e_{i}$ is adjacent to the pathos vertex $P_{i}$ to form a pendent vertex. Since in top semirelib graph of a tree, one path of pathos is adjacent to at least other pathos we have degree of a pathos vertex is at least two. Hence by the definition, $\mathrm{T}_{\mathrm{ps}}(\mathrm{T})$ is separable.

In the following theorem we obtain the condition for the planarity on top semirelib graph of a tree.

\section{Theorem 3.7:}

For any non trivial tree $\mathrm{T}$, the $\mathrm{T} \mathrm{p}(\mathrm{T})$ is planar if and only if $\mathrm{T}$ is a path $\mathrm{P}_{\mathrm{n}}$ or $\mathrm{T}$ is $\mathrm{K}_{1,3}$.

Proof: Suppose $T_{p}(T)$ is planar. Assume that $\exists_{V_{i}} \in T$ such that $\operatorname{deg}_{\mathrm{i}} \geq 3$ and $\mathrm{T}$ is not $\mathrm{K}_{1,3}$. Suppose $\operatorname{deg} v_{\mathrm{i}}=3$ and $\mathrm{e}_{1}, \mathrm{e}_{2}, \mathrm{e}_{3}$ are the edges incident to $\mathrm{v}_{\mathrm{i}}$. By the definition of line graph, $\mathrm{e}_{1}, \mathrm{e}_{2}, \mathrm{e}_{3}$ form $\mathrm{K}_{3}$ as an induced subgraph. In $T_{p s}(T)$, the region vertex $r_{i}$ is adjacent with all vertices of $\mathrm{L}(\mathrm{T})$ to form $\mathrm{K}_{4}$ as an induced subgraph. Further the corresponding block vertices $b_{1}, b_{2}, b_{3}, \ldots$, $\mathrm{b}_{\mathrm{n}-1}$ of of blocks $\mathrm{B}_{1}, \mathrm{~B}_{2}, \mathrm{~B}_{3}, \ldots, \mathrm{B}_{\mathrm{n}}$ in $\mathrm{T}$ are adjacent to vertices of $K_{4}$ and the pathos vertices are adjacent to the edges lies on the paths of $\mathrm{T}$, which forms graph homeomorphic to $\mathrm{K}_{5}$. By the Theorem 7.2.3, it is non planar, a contradiction.

Conversely, Suppose $\mathrm{T}$ is a path $\mathrm{P}_{\mathrm{n}}$. By the definition of line graph $L\left[P_{n}\right]=P_{n-1}$. In $T_{p s}(T)$, the region vertex $r_{i}$ is adjacent to all vertices of $\mathrm{L}(\mathrm{G})$. Further, by the lemma 7. 2.2, the blocks $b_{1}, b_{2}, b_{3}, \ldots, b_{n}$ of $T$ with $n$ vertices such that $\mathrm{b}_{1}=\mathrm{e}_{1}, \mathrm{~b}_{2}=\mathrm{e}_{2}, \ldots, \mathrm{b}_{\mathrm{n}-1}=\mathrm{e}_{\mathrm{n}-1}$ becomes $\mathrm{p}-1$ pendant vertices. The pathos vertices have degree at most three. The pathos vertices $\mathrm{p}_{1}, \mathrm{p}_{2}, \mathrm{p}_{3}$ are adjacent to each other. Clearly, $T_{p s}(T)$ is planar.

In the following theorem we obtain the condition for the outer planarity on Top semirelib graph of a tree.

\section{Theorem 3.8:}

For any non trivial tree $\mathrm{T}, \mathrm{T}_{\mathrm{ps}}(\mathrm{T})$ is outer planar if and only if $T$ is a path $P_{n}, n \leq 3$.

Proof: Proof follows from the theorem 7.2.5.

In the following theorem we obtain the condition for the non eulerian on Top semirelib graph of a tree

\section{Theorem 3.9:}

For any tree $\mathrm{T}, \mathrm{P}_{\mathrm{s}}(\mathrm{G})$ is always non Eulerian.

Proof: Suppose $\mathrm{T}$ is a tree. In a tree each edge is a block and hence $\mathrm{b}_{1}=\mathrm{e}_{1}, \mathrm{~b}_{2}=\mathrm{e}_{2}, \ldots, \mathrm{b}_{\mathrm{n}-1}=\mathrm{e}_{\mathrm{n}-1}, \forall \mathrm{e}_{\mathrm{n}-1}$ $\in \mathrm{E}(\mathrm{T})$ and $\forall \mathrm{b}_{\mathrm{n}-1} \in \mathrm{V}\left[\mathrm{P}_{\mathrm{s}}(\mathrm{T})\right]$. In $\mathrm{P}_{\mathrm{s}}(\mathrm{T})$, the block vertex $b_{i}$ is always a pendent vertex, which is non Eulerian.

In the following theorem we obtain the condition for the hamiltonian on Top semirelib graph of a tree.

\section{Theorem 3.10:}

For any tree $\mathrm{T}, \mathrm{T}_{\mathrm{ps}}(\mathrm{T})$ is non hamiltonian.

Proof: Suppose $\mathrm{T}$ is a tree. In a tree each edge is a block and hence $\mathrm{b}_{1}=\mathrm{e}_{1}, \mathrm{~b}_{2}=\mathrm{e}_{2}, \ldots, \mathrm{b}_{\mathrm{n}-1}=\mathrm{e}_{\mathrm{n}-1}, \forall \mathrm{e}_{\mathrm{n}-1}$ $\in \mathrm{E}(\mathrm{T})$ and $\forall \mathrm{b}_{\mathrm{n}-1} \in \mathrm{V}\left[\mathrm{P}_{\mathrm{s}}(\mathrm{T})\right]$. In $\mathrm{P}_{\mathrm{s}}(\mathrm{T})$, the block vertex $b_{i}$ is always a pendent vertex, which is non hamiltonian.

\section{Conclusions}

The concept of top semirelib graph of a planar graph is introduced. We presented a characterization of graphs, whose top semirelib graphs are planar, outer planar, eulerian, hamiltonian . 


\section{References}

[1] Chartrand G, Geller, D and Hedetniemi, S. J. Combinatorial Theory (1971)10:12.

[2] Harary F., Graph theory, Addition - Weseley Reading .Mass.(1969), pp 34 and 107.

[3] Harary, F., Annals of New York Academy of Science,(1975),175:198.
[4] Kulli V R ., On Minimally Non- Outer Planar Graphs, Proceeding of the Indian National Science Academy, Vol.41, Part A, No.3 (1975), pp 275 280.

[5] Venkanagouda M Goudar., K B Manjunatha. "Pathos Semirelib Graph of a Tree", in the Global Journal of Mathematical Sciences: Theory and Practical Vol 5, No.2,(2013),pp1-6. 